\title{
The Formation and Evolution of Star Clusters and Galaxies
}

\author{
Stephen E. Zepf \\ Dept. of Physics and Astronomy, Michigan State University, East \\ Lansing, MI 48824, USA
}

\begin{abstract}
This paper addresses the questions of what we have learned about how and when dense star clusters form, and what studies of star clusters have revealed about galaxy formation and evolution. One important observation is that globular clusters are observed to form in galaxy mergers and starbursts in the local universe, which both provides constraints on models of globular cluster formation, and suggests that similar physical conditions existed when most early-type galaxies and their globular clusters formed in the past. A second important observation is that globular cluster systems typically have bimodal color distributions. This was predicted by merger models, and indicates an episodic formation history for elliptical galaxies. A third and very recent result is the discovery of large populations of intermediate age globular clusters in several elliptical galaxies through the use of optical to near-infrared colors. These provide an important link between young cluster systems observed in starbursts and mergers and old cluster systems. This continuum of ages of the metal-rich globular cluster systems also indicates that there is no special age or epoch for the formation of the metal-rich globular clusters, which comprise about half of the cluster population. The paper concludes with a brief discussion of recent results on the globular cluster - low-mass X-ray binary connection.
\end{abstract}

\section{Globular Cluster Formation}

A natural starting point for the discussion of galaxies and globular cluster systems is the formation of globular clusters (GCs). Any model of the formation of globular cluster systems and their host galaxies that does not include a consideration of how the globular clusters themselves form is necessarily incomplete. Fortunately, nature has provided us nearby examples of GC formation, most dramatically revealed in HST images (e.g. Whitmore et al. 1999, Zepf et al. 1999). The formation of globular clusters in galaxy mergers was predicted by Ashman \& Zepf (1992) and Schweizer (1987) and then confirmed by many subsequent HST observations. Moreover, as discussed at this meeting by Bruce Elmegreen, these observations are providing an important testing ground for theoretical work on GC formation (e.g. Elmegreen 2002, Ashman \& Zepf 2001).

\section{Key Properties of Globular Cluster Systems}

A second key point is the bimodality typical of the color distributions of earlytype galaxies. The bimodality of early-type galaxy globular cluster systems was first discovered about ten years ago (Zepf \& Ashman 1993), and has now been confirmed to be typical by extensive studies of archival HST data (Kundu \& 
Whitmore 2001, Larsen et al. 2001). The data show that roughly half of the GCs are blue (metal-poor) and half are red (metal-rich, i.e. very roughly solar or somewhat less). There is little or no trend of the red/blue ratio with galaxy luminosity or globular cluster number (e.g. Rhode \& Zepf 2003).

The observed bimodality in the cluster systems of elliptical galaxies has important implications for the formation of globular clusters and galaxies. Firstly, bimodality was predicted by Ashman \& Zepf (1992) to result from elliptical galaxy formation by mergers of disk galaxies, mostly at $z \gtrsim 1$, and so the observation was in agreement with an earlier theoretical prediction. Moreover, regardless of the specific model, bimodality requires an episodic formation history for elliptical galaxies, and not a single formation event. Secondly, most of these events also have to occur at least $\sim 8 \mathrm{Gyr}$ ago in order for the metal-rich GCs to become red enough to produce the color bimodality from the metallicity bimodality. However, even if most of the major formation events take place at $z \gtrsim 1$, some will take place more recently, and to make the picture complete, we should be able to identify and characterize these. This has recently been achieved, as discussed in the following section.

\section{Intermediate Age Globular Cluster Systems}

The discovery of intermediate age globular cluster systems in elliptical galaxies is important because it links the young systems observed in mergers to the traditional old GC systems. A key observational advance has been the use of optical to near-infrared colors to break the age-metallicity degeneracy to identify intermediate age, $\sim$ solar metallicity globular clusters (which are effectively indistinguishable from lower metallicity, older clusters in optical colors alone).

The breakthrough in identifying intermediate age clusters came in two ways. Puzia et al. (2002) discovered a large population of intermediate age globular clusters in the otherwise fairly normal elliptical galaxy NGC 4365. Subsequent spectroscopy of a subset of the Puzia et al. (2002) clusters confirmed the effectiveness of their optical to near-infrared technique (Larsen et al. 2003). While Puzia et al. (2002) demonstrated that some quiescent ellipticals had a major formation event in their not so distant past, Goudfrooij et al. (2001) used similar techniques to identify a major intermediate-age globular cluster population in the disturbed galaxy NGC 1316. More recently, Hempel et al. (2003) found a significant intermediate-age cluster population in NGC 5846. Both Puzia et al. (2002) and Hempel et al. (2001) also find galaxies without intermediate age populations, as expected since the common bimodal systems are probably not intermediate-age. However, the presence of intermediate age systems shows that the age distribution of cluster systems is continuous from very young to very old, with all ages present. The formation of these clusters traces out the starburst histories of their host ellipticals, which appears to be complex and to occur over a significant range of times.

\section{Globular Cluster - Low-Mass X-Ray Binary Connection}

Since this is a dynamics session, and GCs are fertile ground for the study of stellar dynamics, it is relevant to note that the dynamical interactions between 
stars play an important role in understanding X-ray emission from elliptical galaxies. This is because roughly half of all X-ray binaries seen in Chandra images are located in GCs (see upcoming review by Verbunt \& Lewin 2003 and references therein). Moreover, the dynamical formation of X-ray binaries in GCs may be the dominant process in early-type galaxies, since sources not now in GCs may have been formed there and ejected or been in clusters that were disrupted (e.g. Maccarone, Kundu, \& Zepf 2003). The study of the GCs hosting low-mass X-ray binaries also provides constraints on the physics of X-ray binary formation and evolution. In particular, there is a strong metallicity dependence and no strong age dependence (Kundu et al. 2003 and references therein).

Acknowledgments. I thank my many collaborators on the projects described here, and also my postdocs and graduate students Arunav Kundu, Katherine Rhode, Enrico Vesperini, Gilles Bergond, and Chris Waters. Financial support is acknowledged from LSTA grant NAG5-11319 and Chandra and HST programs.

\section{References}

Ashman, K.M., \& Zepf, S.E. 1992, ApJ, 384, 50

Ashman, K.M., \& Zepf, S.E. 2001, AJ, 122, 1888

Elmegreen, B.G. 2002, ApJ, 577, 206

Goudfrooij, P., et al. 2001, MNRAS, 322, 643

Hempel, M., et al. 2003, A\&A, 405, 487

Kundu, A., \& Whitmore, B.C. 2001, AJ, 121, 2950

Kundu, A., Maccarone, T.J., Zepf, S.E., \& Puzia, T.H. 2003, ApJL, 589, 81

Larsen, S.S., et al. 2001, AJ, 121, 2974

Larsen, S.S., et al. 2003, ApJ, 585, 767

Maccarone, T.J., Kundu, A., Zepf, S.E. 2003, ApJ, 586, 814

Puzia, T.H., Zepf, S.E., Kissler-Patig, M., Hilker, M., Minniti, D., \& Goudfrooij, P. 2002, A\&A, 391, 453

Rhode, K.L., \& Zepf, S.E. 2003, AJ, 126, 2307

Schweizer, F. 1987, in Nearly Normal Galaxies, ed. S.M. Faber (Berlin: Springer), 18

Verbunt, F. \& Lewin, W.H.G. 2003, in preparation

Whitmore, B.C., Zhang, Q., Leitherer, C. Fall, S.M., Schweizer, F., \& Miller, B.W. 1999, AJ, 118, 1551

Zepf, S.E., \& Ashman, K.M. 1993, MNRAS, 264, 611

Zepf, S.E., Ashman, K.M., English, J., Freeman, K.C., \& Sharples, R.M. 1999, AJ, 118, 752 